ДЕФИЦИТ ГОРМОНА РОСТА У ЛИЦ, ПЕРЕНЕСШИХ ХИМИОЛУЧЕВУЮ ТЕРАПИЮ ЗЛОКАЧЕСТВЕННЫХ ОПУХОЛЕЙ ГОЛОВНОГО МОЗГА И ОСТРОГО ЛИМФОБЛАСТНОГО ЛЕЙКОЗА В ДЕТСТВЕ

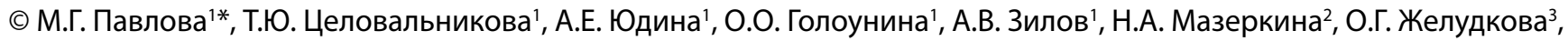
В.В. Фадеев ${ }^{1}$

'Первый Московский государственный медицинский университет им. И.М. Сеченова (Сеченовский Университет), Москва, Россия

${ }^{2}$ Национальный медицинский исследовательский центр нейрохирургии им. академика Н.Н. Бурденко, Москва, Россия ${ }^{3}$ Научно-практический центр специализированной медицинской помощи детям им. В.Ф. Войно-Ясенецкого, Москва, Россия

Благодаря внедрению в клиническую практику новых методов диагностики и стандартизированных протоколов лечения число пациентов, перенесших в детстве онкологическое заболевание и успешно преодолевших барьер пятилетней выживаемости, увеличивается с каждым годом. Противоопухолевая терапия неизбежно сопровождается развитием тяжелых отдаленных последствий, в том числе со стороны эндокринной системы, среди которых недостаточность гормона роста встречается чаще других. Низкий конечный рост затрудняет психологическую и социальную адаптацию пациентов, снижает качество их жизни. Влияние заместительной терапии препаратами гормона роста на физиологические процессы и состояние здоровья в целом вызывает большой интерес исследователей. В ряде работ продемонстрировано потенцирующее воздействие гормона роста и инсулиноподобного фактора роста-1 на рост и пролиферацию клеток. В этой связи вопрос об увеличении риска рецидива заболевания или развития вторичных опухолей у пациентов, перенесших онкологическое заболевание, вызывает много споров и является предметом непрерывного изучения. В настоящем обзоре обобщены имеющиеся в литературе данные о патогенезе и распространенности дефицита гормона роста, развивающегося после химиолучевой терапии злокачественных новообразований в детстве, в частности после лечения опухолей головного мозга и острого лимфобластного лейкоза. Обсуждаются имеющиеся проблемы диагностики дефицита гормона роста, безопасность заместительной терапии дефицита гормона роста у детей и взрослых.

КЛЮЧЕВЫЕ СЛОВА: гормон роста; дефичит гормона роста; лучевая терапия; заместительная терапия дефицита гормона роста; злокачественные новообразования; опухоли головного мозга; острый лимфобластный лейкоз.

\title{
GROWTH HORMONE DEFICIENCY IN CHILDHOOD BRAIN TUMORS AND ACUTE LYMPHOBLASTIC LEUKEMIA SURVIVORS
}

(c) Maria G. Pavlova ${ }^{1 *}$, Tatiana Y. Tselovalnikova' ${ }^{1}$ Alla E. Yudina', Olga O. Golounina', Alexey V. Zilov'1, Nadezhda A. Mazerkina², Olga G. Zheludkova ${ }^{3}$, Valentin V. Fadeev ${ }^{1}$

'I.M. Sechenov First Moscow State Medical University (Sechenov University), Moscow, Russia

${ }^{2}$ N.N. Burdenko national medical research center of neurosurgery, Moscow, Russia

${ }^{3}$ Voino-Yasenetsky Krasnoyarsk State Medical University, Moscow, Russia

Thanks to modern treatment protocols, childhood cancer survivors (CCS) are a very fast-growing population nowadays. Cancer therapy inevitably leads to different late adverse effects, where endocrine disorders are highly prevalent, including growth hormone deficiency (GHD) which is the most common endocrine outcome after cancer treatment in childhood and contributes to impaired growth. Short stature is a big issue, which leads to problems in psychological and social adaptation of patients and reduces their quality of life. Impact of GH treatment on various physiological processes and global outcome of CCS is of great interest. Several studies have demonstrated an influence of GH and IGF-1 on the development/tumour growth, cell proliferation. In this regard, the issue of increasing the risk of cancer recurrence and/or the development of secondary neoplasms in CCS, causes a lot of controversy and is the subject of continuous evaluation. In this review, we went through the available data on the prevalence and pathogenesis of GHD following chemo- and radiotherapy, in particular after treatment of brain tumors and acute lymphoblastic leukaemia in childhood. In addition, here we discuss the existing problems in the diagnosis of GHD, the safety of GH replacement therapy, as well as the treatment algorithm of the GHD in adults.

KEYWORDS: growth hormone; growth hormone deficiency; cancer; radiation therapy; growth hormone replacement therapy; malignant neoplasms; brain tumors; acute lymphoblastic leukaemia. 


\section{ВВЕДЕНИЕ}

В последние десятилетия во всем мире наблюдается неуклонный рост злокачественных новообразований (3НО) у детей и подростков [1]. Внедрение в клиническую практику новых методов диагностики и стандартизированных протоколов лечения способствовало значительному снижению смертности и увеличению выживаемости лиц с онкологическими заболеваниями. В 2019 г. в Российской Федерации от ЗНО умерли 842 ребенка в возрасте 0-17 лет (2,82 случая на 100000 детского населения), что существенно ниже 1994 г. - 1888 детей (5,9 случая на 100 000) [2]. По данным American Cancer Society, 5-летняя выживаемость при всех видах злокачественных опухолей, диагностированных у детей и подростков (в возрасте 0-19 лет) в США, с 2010 по 2016 гг. выросла до 85\% по сравнению с 63\% среди тех, кому диагноз был поставлен в середине 1970-х гг. [1].

В структуре $3 \mathrm{HO}$ у детей первое место занимают гемобластозы, удельный вес которых в 2017 г. составил 50\%, далее - опухоли головного мозга (ОГМ) и других отделов центральной нервной системы (ЦНС) (19\%), нейробластомы, опухоли почек, костей и мягких тканей [3]. Следует отметить, что опухоли ЦНС занимают лидирующую позицию среди всех солидных опухолей детского возраста.

Лечение онкологических заболеваний у детей предусматривает комплексный подход, включающий хирургическое удаление опухоли (для солидных опухолей), полихимиотерапию (ПХТ) и лучевую терапию (ЛТ), в частности краниальное (КО) или краниоспинальное облучение (КСО). Однако данные протоколы, наряду с увеличением выживаемости, неизбежно приводят к развитию различных отдаленных последствий, в том числе и со стороны эндокринной системы. Наиболее частым эндокринным нарушением является недостаточность гормона роста (ГР), относительный риск (ОР) развития которой у пациентов, перенесших лечение ОГМ в детстве, выше в 277,8 раза по сравнению со здоровой популяцией [4]. Дефицит ГР (ДГР) приводит к низкому конечному росту, что существенно затрудняет психологическую и социальную адаптацию пациентов и снижает качество их жизни. Необходимо отметить, что не только ДГР, но и некомпенсированный гипотиреоз, преждевременное половое развитие вносят существенный вклад в замедление ростовых процессов у лиц, перенесших противоопухолевое лечение. Кроме того, повреждение ростовых зон позвоночника в результате КСО в сочетании с ПХТ, прием супрафизиологических доз глюкокортикоидов, недостаточное питание и мальабсорбция во время проведения химиолучевого лечения также влекут за собой нарушение ростовых процессов и отставание в росте.

В настоящем обзоре подробно представлены потенциальные механизмы развития радиоиндуцированного ДГР, основные причины и факторы риска нарушений роста, а также положительные и отрицательные стороны заместительной терапии ДГР и ее безопасность у лиц, перенесших в детстве химиолучевое лечение 3НО, в частности ОГМ и острого лимфобластного лейкоза.

\section{РАСПРОСТРАНЕННОСТЬ И ПАТОГЕНЕЗ РАДИОИНДУЦИРОВАННОГО ДЕФИЦИТА ГОРМОНА РОСТА У ПАЦИЕНТОВ, ПЕРЕНЕСШИХ ХИМИОЛУЧЕВУЮ ТЕРАПИЮ В ДЕТСТВЕ}

KCO, широко применяемое в лечении ОГМ и острого лимфобластного лейкоза, или ЛТ всего тела в процессе подготовки к трансплантации костного мозга неизбежно приводят к гипоталамо-гипофизарной дисфункции (гипопитуитаризму) и множественным клиническим проявлениям дефицита гипофизарных гормонов. Выраженность гормонального дефицита и степень тяжести во многом зависят от суммарной дозы и области облучения, возраста пациента на момент лечения [5]. Известно, что клетки гипофиза обладают различной радиочувствительностью. Так, соматотрофы наиболее восприимчивы к воздействию Лт, чуть менее чувствительны клетки, секретирующие лютеинизирующий (ЛГ) и фолликулостимулирующий (ФСГ) гормоны, в то время как кортикотрофы и тиреотрофы наиболее устойчивы к влиянию облучения. В исследованиях in vitro ЛТ в дозе 3 Гр снижает секрецию ГР и пролактина, в то время как продукция тиреотропного гормона (ТТГ) остается неизменной даже в случае воздействия ЛТ в дозах более 10 Гр [5].

В свою очередь, КО, применяемое при лечении злокачественных опухолей головного мозга и вовлекающее гипоталамо-гипофизарную область, также сопровождается развитием недостаточности ГP [4]. S. Uday и соавт. [6] диагностировали ДГР у 97\% пациентов после лечения медуллобластом, развившийся у подавляющего большинства детей в течение первых 2 лет с момента окончания лечения (1,7 года (0,7-15 лет)). По разным данным, при воздействии дозы 30-50 Гр частота развития недостаточности ГР может достигать 50-100\%. Однако снижение секреции ГР может наблюдаться и при воздействии более низких доз ЛТ, например, при КО, применяемом при остром лимфобластном лейкозе для профилактики нейролейкоза. M. Mirouliaei и соавт. [7] оценили распространенность эндокринных последствий КО при остром лимфобластном лейкозе у детей. В исследование были включены 27 детей с острым лимфобластным лейкозом, получивших КО в дозе 18-24 Гр. Оценка уровня гормонов проводилась до проведения ЛТ, через 6 мес после ее завершения и для отдельных показателей ежегодно. По результатам исследования, ДГР, диагностированный при помощи теста с клонидином, был выявлен у 44\% пациентов, из них у половины данная патология развилась менее чем через 1 год после Лт. Только у 2 пациентов наблюдалось увеличение уровня ТТГ более 5 мМЕд/мл. В течение 9-летнего периода наблюдения не было зарегистрировано нарушений в оси гипоталамус-гипофиз-надпочечники: уровни адренокортикотропного гормона (АКТГ) и кортизола оставались в пределах референсного диапазона. Уровень половых гормонов также был в пределах нормы. У 18,5\% пациентов, включенных в исследование, было выявлено ожирение.

ЛТ негативно влияет на ростовые пластины позвоночника, вызывая их деструкцию, что приводит к нарушению процессов спинального роста и, как следствие, к низкому конечному росту. По данным литературы, в течение первых лет после ЛТ у пациентов, получивших КСО, отмечается более низкий рост, чем у тех, кто получил только КО [8]. 
В исследовании J. Olshan и соавт. [8] ДГР развивался спустя 2 года после завершения противоопухолевой терапии, поэтому задержка роста в первые 2 года после окончания лечения у пациентов, получивших КСО, была ассоциирована не с ДГР, а в основном с нарушением спинального роста, вызванного воздействием КСО. Таким образом, не только КО, но и КСО способствует снижению роста.

Время манифестации радиоиндуцированной недостаточности ГР и ее тяжесть в первую очередь зависят от общей дозы облучения, фракционной дозы, а также времени между фракциями, отведенного для восстановления тканей. Считается, что проведение ЛТ в течение длительного периода, но малыми фракционными дозами менее пагубно для организма в сравнении с воздействием больших фракционных доз за короткий период. При воздействии доз менее 40 Гр зачастую развивается изолированный ДГР, тогда как применение больших доз приводит к недостаточности и других гормонов гипофиза [9]. От дозы облучения зависит и тип нарушения секреции ГР. М. Jarfelt и соавт. [10] оценивали суточную спонтанную секрецию ГР у пациентов после КО по поводу острого лимфобластного лейкоза в детстве. Несмотря на незначительный отрицательный эффект на конечный рост (только 1 из 35 пациентов после КО не достиг целевого роста), у 79\% молодых взрослых после КО в низких дозах в детстве было выявлено снижение спонтанной секреции ГР. При облучении более 27 Гр у 65\% пациентов отмечалось снижение как спонтанной, так и стимулированной секреции, а при воздействии дозы свыше 30 Гр снижение стимулированной секреции наблюдается практически в $100 \%$ случаев.

На сегодняшний день патофизиология радиоиндуцированного ДГР до конца не ясна. Ранее считалось, что ЛТ в основном поражает гипоталамус. По мнению ряда ученых, при КО в дозе менее 40 Гр ДГР развивается в результате поражения гипоталамуса, поскольку он более радиочувствителен, чем гипофиз, а воздействие доз выше 50 Гр приводит к повреждению гипофиза [11]. Эта гипотеза основана на относительно сохранном или нормальном выбросе ГР в ответ на прямую стимуляцию гипофиза экзогенным рилизинг-гормоном ГР (рГГР) по сравнению с ответом на стимуляцию в других провокационных пробах, особенно в пробе с инсулиновой гипогликемией (ИГГ). Однако последние данные свидетельствуют о прямом повреждающем воздействии ЛТ в дозах менее 40 Гр на гипофиз, а при облучении в дозах более 40 Гр поражаются как гипофиз, так и гипоталамус [12].

Секреция ГР имеет импульсный характер. Длительность такого секреторного «импульса» варьирует от 2 до 6 ч, при этом амплитуда может меняться в пределах 5-20 мкг/л. Иногда наблюдается так называемая «залповая» секреция, когда один пик включает ряд последовательных импульсов продолжительностью 30-40 мин каждый. При исследовании спонтанного суточного ритма было показано, что уровень ГР в крови зависит от возраста, пола и стадии полового развития и колеблется от 0,02 до 0,06 ME/кг/сут. Для оценки спонтанной секреции ГР проводится анализ профиля ГР (количество импульсов, их амплитуда, суммарная концентрация ГР) в течение 24 ч с забором крови каждые 20 мин. В исследовании K. Darzy и соавт. [13] было показано, что у пациентов с дефицитом ГР после облучения по поводу вне- гипофизарных ОГМ в детском возрасте при контроле суточного ритма ГР отмечается статистически значимое снижение во всех амплитуд-связанных измерениях (средний уровень ГР, максимальный пик ГР, средняя высота пика, среднее значение «импульса»). В то же время авторы не выявили значимых отличий в частоте импульсов секреции ГР, длительности импульсов и межимпульсных интервалах по сравнению с контрольной группой.

Вероятность развития недостаточности ГР во многом зависит от возраста на момент лечения. Ранние наблюдения, посвященные изучению частоты дефицита ГР, показали, что молодой возраст (менее 18 лет) повышает уязвимость перед радиационным воздействием. Изолированный ДГР после КО в дозе 18-24 Гр чаще развивается у детей, чем у взрослых, поскольку гипоталамогипофизарная область детей более радиочувствительна по сравнению с таковой у взрослых [14]. ДГР часто выявляют у детей, получивших облучение всего тела, и практически никогда у взрослых, подвергшихся ЛТ в сопоставимой дозе. У детей младшего возраста с острым лимфобластным лейкозом после КО риск развития недостаточности ГР значимо выше, чем у пациентов в пубертате [14]. По данным N. Samaan и соавт. [15], из 166 пациентов в возрасте от 6 до 80 лет, которым было проведено КО в высоких дозах по поводу опухолей головы и шеи, у детей до 15 лет недостаточность ГР вскоре после ЛТ регистрировалась чаще, чем у пожилых пациентов. Однако в старшей возрастной группе с более высокой частотой отмечался дефицит АКТГ и ЛГ.

\section{ДИАГНОСТИКА ДЕФИЦИТА ГОРМОНА РОСТА}

Учитывая высокую вероятность развития недостаточности ГР после ЛТ, всем детям, подвергшимся облучению, необходимо регулярно (каждые 3-6 мес) измерять рост стоя и сидя. Рост менее -2 SDS, снижение скорости роста (SDS скорости роста -1 и менее и/или нарастание разницы между SDS роста ребенка и SDS целевого роста) показания для оценки секреции ГР.

Инсулиноподобный фактор роста-1 (ИФР-1) и белок-3, связывающий инсулиноподобные факторы роста (ИФРСБ-3), - интегральные показатели секреции ГР, используемые в качестве скрининговых параметров. Однако зачастую их значения находятся в пределах референсного диапазона даже у лиц с доказанной недостаточностью ГР $[16,17]$, Таким образом, ни один из этих показателей по отдельности не является надежным маркером ДГР.

Ключевую роль в диагностике недостаточности ГР играют провокационные пробы. Измерение суточной спонтанной секреции ГР оправдано в случае, когда наблюдается субнормальная скорость роста наряду с нормальными результатами стимуляционных тестов [18]. Подобная ситуация отражает легкую степень повреждения соматотрофов в результате воздействия малых доз облучения (20-30 Гр) наряду с неадекватной функцией гипоталамуса (нейросекреторная дисфункция ГР). Однако к данному исследованию в клинической практике прибегают крайне редко ввиду его высокой стоимости и малодоступности [9].

Стимуляционные тесты для диагностики недостаточности ГР основаны на способности различных фармакологических препаратов повышать секрецию ГР 
гипофизом. К настоящему времени опубликовано несколько международных консенсусов по диагностике дефицита ГР у взрослых [19-21], которые рекомендуют применение различных стимуляционных тестов. Интересно, что авторы консенсусов предлагают использовать опубликованные в ряде исследований пороговые значения ГР в тестах, одновременно с этим высказывая сомнения относительно сопоставимости уровней ГР, поскольку в разных тестах они определялись разными методами. Таким образом, применяемые на сегодняшний день отрезные точки для ГР в стимуляционных тестах имеют слабую доказательную базу, что в основном обусловлено несоответствием между концентрациями ГР, измеренными разными методиками. При использовании различных тест-систем в одних и тех же образцах крови выявляется широкая вариабельность значений ГР. В результате у одного и того же человека можно получить значения, соответствующие как нормальной секреции ГР, так и ДГР [22]. С появлением новых методов, основанных на применении моноклональных антител (АТ), а не поликлональных, как это было раньше, расхождения между уровнями ГР, определенными разными тест-системами, усугубились еще больше.

В измерении ГР существует несколько проблем. Во-первых, ГР - это не гомогенная молекула. Циркулирующий в крови ГР представлен большим числом молекулярных изоформ: есть и мультимеры, и димеры [23]. Последние могут встречаться в виде гетеро- или гомодимеров. Ввиду того, что специфические АТ, используемые в тест-системах, распознают только специфические эпитопы, присутствующие на поверхности антигена (АГ), каждое АТ определяет только конкретный спектр изоформ. Вероятнее всего, этот спектр для поликлональной антисыворотки шире, чем для моноклональной, что может объяснять разницу между измерениями ГР при помощи этих двух разных методов [24]. Второй фактор, влияющий на расхождение между результатами измерения ГР, - это ГР-связывающий белок [25]. 50\% циркулирующего ГР находится в связанном состоянии, поэтому он может быть недоступен для определения AT, что может привести к недооценке концентрации ГР. Третий фактор - это метод калибровки тест-системы. Ранее для определения ГР в качестве калибратора широко применяли ГР, выделенный из ткани гипофиза (International Standard (IS) 66/217 от 1969 г., IS 80/505 от 1982 г.). Однако такой ГР содержит разные изоформы и примеси. В этой связи для калибровки было предложено использовать более «чистый» рекомбинантный ГP (IS 98/574). Очевидно, что изменение стандарта калибровки оказало основное влияние на абсолютную концентрацию ГР [26] — при использовании IS 98/574

Таблица 1. Диагностика дефицита гормона роста у взрослых концентрация ГР в целом ниже, чем была при использовании IS 66/217 и IS 80/505. Помимо указанных факторов, перевод в другие единицы измерения также создает дополнительные сложности в сопоставлении результатов ГР. Одни лаборатории представляют данные в Ед/л, в то время как другие - в мкг/л, и во многих случаях критерии перевода одних единиц в другие неясны. Как результат, многообразие используемых методов калибровки, а также единиц измерения приводит к путанице и сложностям при публикации данных клинических исследований. С целью улучшения сопоставимости измерений ГР, проведенных в разных учреждениях, некоторые исследователи предложили повторно измерять ГР централизованно одним конкретным методом [27], что невозможно в рутинной клинической практике. Ряд клинических рекомендаций $[19,28]$ подчеркивает необходимость использования «метод-специфических отрезных точек», но для большинства применяемых сегодня тест-систем какой-либо справочной информации по этому вопросу крайне мало, в связи с чем на сегодняшний день это также остается невозможным.

Международная группа специалистов по изучению ГР (Growth Hormone Research Society) [29] предложила возможные пути стандартизации методов его определения. Применение только рекомбинантного ГР в качестве калибратора (IS 98/574) и представление данных в едином формате, а именно в мкг/л - первые важные шаги в решении данного вопроса. Growth Hormone Research Society также опубликовало в своих алгоритмах диагностики дефицита ГР у взрослых рекомендацию по применению в качестве калибратора только рекомбинантного ГР (IS 98/574) [19]. При применении в калибровке стандарта IS 98/574 вариабельность в уровне ГР между разными лабораториями снизилась с 35 до 20\% [26].

Проба с ИГГ выступает золотым стандартом в диагностике недостаточности ГР как у детей, так и у взрослых [19-21] (табл. 1). Одно из преимуществ данного теста - возможность оценить секреторную функцию как гипоталамуса, так и гипофиза, в связи с чем его можно применять для диагностики дефицита ГР, вызванного как поражением гипоталамуса, так и гипофиза, в частности после КО. С другой стороны, проба с ИГГ имеет и ряд отрицательных сторон: она противопоказана пациентам с судорожным синдромом, ишемической болезнью сердца и сахарным диабетом, в большинстве случаев сопровождается неприятными ощущениями. Кроме того, она имеет низкую воспроизводимость. Так, у здоровых добровольцев, которым тест с ИГГ проводился несколько раз, в разные дни пик ГР отличался в 6 раз, независимо от степени гипогликемии [30].

\section{Growth Hormone Research Society (2019)}

\section{American Association of Clinical Endocrinologists (2009)}

The Endocrine Society (2011)

\section{Проба с инсулиновой гипогликемией} Проба с глюкагоном

ГР $<3$ мкг/л $\quad$ ГР $<5$ мкг/л

ГР $<3$ мкг/л
$Г \mathrm{P}<5$ мкг/л

Примечание: ГР - гормон роста. 
В ряде зарубежных исследований, включавших проведение стимуляционных тестов у здоровыхлиц, в частности пробы с ИГГ, регистрировались ложноположительные результаты у детей и взрослых с нормальным ростом [31, 32]. В одном исследовании авторы связывали низкий пик ГР в пробе с ИГГ с морбидным ожирением и «недостижением» гипогликемии [33]. В других исследованиях подобной зависимости не прослеживалось. В работе H. Markkanen и соавт. [31] оценивалась адекватность использования уровня ГР 3 мкг/л, считающегося минимальной пиковой концентрацией гормона в пробе с инсулином, позволяющей судить о наличии выраженной недостаточности ГР у взрослых пациентов. Этот предел был ранее установлен методом радиоиммунного анализа (РИА) с поликлональными антителами. Было показано, что при использовании современных иммунометрических методов с флуоресцентной или хемилюминесцентной детекцией пик ГР в пробе с ИГГ менее 3 мкг/л выявлялся у 44\% здоровых добровольцев, среди них только 2 человека с избыточным весом не достигли пика и в тесте с рГГР+аргинин. Авторы сделали вывод, что результаты ГР очень сильно зависят от метода его определения. Однако ложноположительные результаты в ИГГ часто получают даже при применении новых тест-систем и современного способа калибровки с более низкой нижней границей для ГР.

Принимая во внимание наличие противопоказаний для пробы с ИГГ, необходим надежный альтернативный тест. Согласно исследованиям, опубликованным с 1998 г., а также мнениям различных экспертных групп, проба с рГГР+аргинином является одной из самых лучших альтернатив пробе с ИГГ (исключая диагностику радиоиндуцированной недостаточности ГР [34]), демонстрируя высокие чувствительность и специфичность. Применение этого теста было возможным до конца 2008 г., однако с тех пор препарат рГГР малодоступен, в связи с чем проведение указанной пробы крайне затруднительно.

Два зарубежных исследования показали, что тест с глюкагоном имеет высокие чувствительность (100\% и 97\%) и специфичность (100\% и $88 \%$ соответственно), надежно выявляя взрослых пациентов с недостаточностью ГР [35, 36]. Кроме того, глюкагон доступен, учитывая его нередкое применение для купирования гипогликемии у пациентов с сахарным диабетом. Тест с глюкагоном достаточно прост в проведении, хорошо переносится пациентами и имеет противопоказания к проведению только среди лиц с недостаточностью питания или тех, кто не принимал пищу в течение более 48 ч. По этим причинам, согласно консенсусам по диагностике ДГР у взрослых, данная проба рекомендована как альтернатива тесту с ИГГ в случае, когда проведение последнего проблематично [20, 21]. Авторы рекомендаций отдельно подчеркивают применение в качестве альтернативного именно теста с глюкагоном, а не c L-DOPA или клонидином [21].

Использование пороговых значений ГР в стимуляционных тестах носит произвольный характер. В ряде исследований проведена оценка пика ГР в различных тестах [30, 37]. Отрезные точки, равные 3 и 5 мкг/л, принятые за критерии диагностики дефицита ГР у взрослых, применялись в клинической практике наиболее часто независимо от фармакологического препарата, который использовался в пробе. Необходимо отметить, что эти пределы были установлены с применением поликлонального
РИА [28]. Нужно ли использовать более низкие пороговые значения с появлением новых, более чувствительных, иммунометрических сэндвич-тестов, до сих пор до конца не определено. Согласно данным одного мультицентрового исследования [33], в котором был использован чувствительный иммунохемилюминесцентный сэндвич-тест, применение порогового значения 5,1 мкг/л в пробе с ИГГ показало достаточную специфичность и чувствительность для диагностики дефицита ГР, что представлено в нескольких диагностических алгоритмах.

Согласно рекомендациям American Association of Clinical Endocrinologists (AACE) 2009 г. [21], The Endocrine Society (США) 2011 г. [20] и Growth Hormone Research Society 2019 г. [19], недостаточность ГР у взрослых пациентов диагностируют при пике выброса ГР в пробе с ИГГ менее 3-5 мкг/л, в пробе с глюкагоном - менее 3 мкг/л. Наряду с этим в указанных диагностических алгоритмах говорится о том, что дефицит 3 и более гормонов гипофиза (в том числе несахарный диабет) в сочетании с ИФР-1, SDS<-2 свидетельствует о наличии недостаточности ГР. В этой ситуации проведение стимуляционных тестов не требуется.

В нашей стране критерии диагностики недостаточности ГР разработаны только для детей. Тотальный ДГР диагностируют при максимальном выбросе ГР на фоне стимуляции менее 7 мкг/л, частичный дефицит - при пике выброса ГР от 7 до 10 мкг/л [38]. Для взрослых пороговые значения не оговорены, что, вероятно, обусловлено тем, что они крайне редко получают заместительную терапию ГР.

\section{ЗАМЕСТИТЕЛЬНАЯ ТЕРАПИЯ ДЕФИЦИТА ГОРМОНА РОСТА}

Недостаточность ГР - один из важнейших факторов снижения линейного роста у детей после КО. Согласно многочисленным данным, заместительная терапия препаратами рекомбинантного ГР (рГР) у таких пациентов может предотвратить возможное снижение темпов роста и способствовать его увеличению. К примеру, конечный рост у больных с медуллобластомой без лечения ГР составляет от $-2,9$ до -5,0 SD [39].

Согласно исследованиям in vitro и in vivo, ГР и ИФР-1 обладают митогенной и антиапоптозной активностью, в связи с чем неизбежно возникает вопрос о влиянии ГР на рост опухоли de novo, а также на рецидив злокачественного образования [40]. По некоторым данным, терапия ГР может увеличивать риск рецидива онкологического заболевания или рост вторичной опухоли у пациентов после лечения 3НО. На вопрос о возможной связи лечения ГР с рецидивом/ростом новой опухоли на сегодняшний день нет однозначного ответа. Данная тема по-прежнему вызывает много споров.

Группой Childhood Cancer Survivor Study (CCSS) было проведено крупное исследование на предмет рецидива онкологического заболевания на фоне терапии препаратами ГР среди более чем 14000 детей, преодолевших барьер 5-летней выживаемости после лечения различных $3 \mathrm{HO}$ [41]. ОР рецидива у лиц, получавших терапию ГP $(\mathrm{n}=361)$, составил 0,83 (95\% доверительный интервал (ДИ) 0,37-1,86) по сравнению с теми, кто не получал заместительную терапию по поводу ДГР [42]. Более того, 
ОР рецидива на фоне терапии ГР не был увеличен ни для одного из отдельных видов 3 НО.

S. Arslanian и соавт. [43] представили результаты применения рГР у детей после лечения ОГМ. Из 34 пациентов 24 получали терапию рГР, у 8 из них (33\%) был отмечен рецидив опухоли. Наряду с этим рецидивы были зафиксированы и у тех, кто не получал лечение рГР (у 3 (30\%) из 10 пациентов). Исследование Н. Chaе и соавт. [44] при длительном наблюдении пациентов с медуллобластомой, которым проводилась терапия рГР, также не выявило рецидивов опухоли. В дополнение к этому в другом исследовании при изучении 545 больных после лечения медуллобластомы не было зарегистрировано ухудшения бессобытийной выживаемости и увеличения риска рецидива опухоли среди 170 лиц, получавших заместительную терапию по поводу ДГР [45].

Некоторые данные свидетельствуют о том, что у пациентов после лечения острого лимфобластного лейкоза, получавших препараты рГР, риск рецидива основного заболевания также не повышается. W. Leung и соавт. [46] исследовали 910 лиц после лечения острого лимфобластного лейкоза в госпитале St. Jude в период 1978-1989 гг., из них 47 человек получили терапию рГР. Медиана длительности ремиссии на момент назначения терапии ГР составила 7 лет (4,3-11,4 года), медиана длительности терапии ГР - 4,5 года (1-8 лет). По результатам анализа полученных данных связь между терапией рГР и рецидивом острого лимфобластного лейкоза не была выявлена. C. Sklar и соавт. [42] обследовали 122 пациента после лечения острого лимфобластного лейкоза, получивших терапию рГР. По результатам анализа ОР рецидива лейкоза у обследованных лиц не увеличивался по сравнению с 4545 пациентами, которые не лечились препаратами рГР.

У пациентов после лечения онкологических заболеваний по сравнению с общей популяцией может отмечаться повышенный риск вторичных неоплазий вследствие ЛТ. Терапия рГР может увеличивать этот риск. Так, по предположению J. Carel и соавт. [47], после терапии рГР риск рецидива злокачественного новообразования может быть невелик, в то время как вероятность развития вторичной опухоли достаточно высока. Однако нельзя достоверно установить, вследствие чего повышается риск вторичных неоплазий — воздействия ЛТ и/или ПХТ или терапии рГР.

Согласно CCSS, пациенты, перенесшие противоопухолевую терапию в детстве, находятся в группе высокого риска вторичных неоплазий независимо от применения препаратов рГР [48]. Наиболее часто встречались рак кожи и рак груди. Три других сообщения CCSS, наоборот, описывают связь между лечением рГР и развитием вторичных неоплазий $[42,49,50]$. По данным C. Sklar [42], ОР любой вторичной опухоли, ассоциированный с воздействием препаратов рГР, повышен в 3,21 раза (95\% ДИ 1,88-5,46).

Согласно проведенному исследованию В. ErgunLongmire и соавт. [49], риск развития вторичных опухолей среди пациентов после лечения онкологических заболеваний выше в 2,15 раза (95\% ДИ 1,3-3,5) у тех, кто получал рГР, по сравнению с теми, кто не лечился рГР (табл. 2). Однако с увеличением длительности наблюдения этот риск снижается. Возможно, это связано с тем, что ГР и ИФР-1 оказывают лишь стимулирующее влияние на рост опухоли, а не инициируют ее рост. По-видимому, терапия ГР не вызывает роста опухоли, но может способствовать и ускорять уже начавшийся канцерогенез. B. Patterson и соавт. ]50] проанализировали данные 12098 детей после лечения $3 \mathrm{HO}$ и выявили, что ОР развития вторичных ОГМ у этих больных составляет 1,0 (95\% ДИ 0,6-1,8) (см. табл. 2).

Н.А. Мазеркина и соавт. [51] провели исследование, включавшее 68 пациентов после лечения различных опухолей головного мозга (35 с краниофарингиомой, 18 с медуллобластомой, 15 с герминативно-клеточными опухолями хиазмально-селлярной области) с недостаточностью ГР, которые получали заместительную терапию рГР в дозах 0,03-0,034 мг/кг/сут. Рецидивы заболевания на фоне заместительной терапии рГР отмечались

Таблица 2. Данные исследований, посвященных изучению риска рецидива новообразований и развития вторичных опухолей у пациентов, перенесших онкологические заболевания, которые получали/не получали терапию препаратами гормона роста

\begin{tabular}{|c|c|c|c|c|c|c|c|c|}
\hline 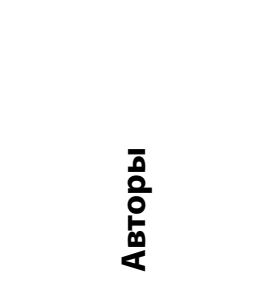 & 동 & 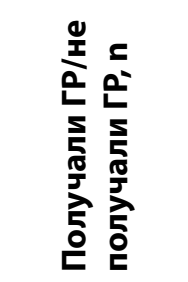 & 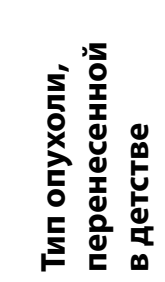 & 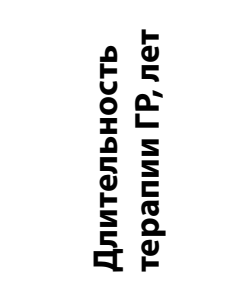 & 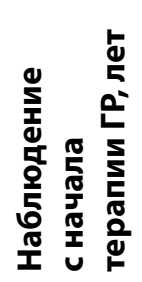 & 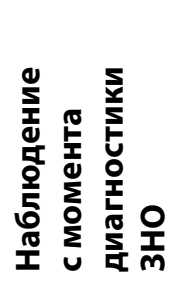 & 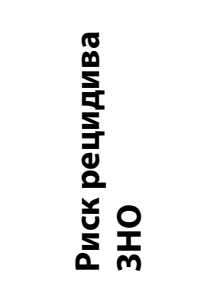 & 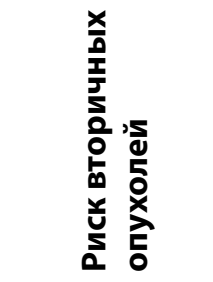 \\
\hline Sklar и соавт. (42) & 2002 & $361 / 12963$ & Все типы & $4,6(0,1-14)$ & 6,2 & $>5$ лет & Не повышен & Повышен \\
\hline $\begin{array}{l}\text { Ergun-Longmir } \\
\text { и соавт. (49) }\end{array}$ & 2006 & $361 / 12963$ & Все типы & $4,6(0,1-14)$ & 8,8 & >5 лет & Не повышен & Повышен \\
\hline $\begin{array}{l}\text { Mackenzie } \\
\text { и соавт. (52) }\end{array}$ & 2011 & $110 / 110$ & ОГМ & $8(1-19)$ & $14,5-15$ & / & Не повышен & Не повышен \\
\hline $\begin{array}{l}\text { Patterson } \\
\text { и соавт. (50) }\end{array}$ & 2014 & $338 / 11760$ & Все типы & не определена & / & 15 лет & / & Не повышен \\
\hline $\begin{array}{l}\text { Brignardello } \\
\text { и соавт. (53) }\end{array}$ & 2015 & $26 / 23$ & Все типы & $3,5(2,7-5)$ & 10 & $>5$ лет & / & Не повышен \\
\hline
\end{tabular}

Примечание: ГР — гормон роста; ЗНО — злокачественные новообразования; ОГМ — опухоли головного мозга. 
у 8 (23\%) из 35 больных с краниофарингиомой, что не отличалось от частоты рецидивов у больных, не получавших рГР. В группах больных с герминомой и медуллобластомой рецидивов заболевания зарегистрировано не было. На фоне терапии рГР не было отмечено каких-либо серьезных нежелательных эффектов.

Лт - хорошо известный фактор риска вторичных опухолей ЦНС. Вторичные глиомы могут развиться в раннем периоде наблюдения, а также во время лечения ГР. Менингиомы чаще всего встречаются в более позднем периоде после ЛТ. Так, случаи вторичных менингиом регистрируют даже спустя 30 лет наблюдения за пациентами. В обновленном анализе CCSS, нацеленном исключительно на изучение риска вторичных опухолей ЦНС, не было выявлено повышения частоты встречаемости менингиом, глиом и других опухолей ЦНС на фоне лечения рГР [50]. Исходя из вышесказанного, по результатам разных исследований, связь развития вторичных опухолей головного мозга с применением рГР не установлена.

Среди пациентов, перенесших химиолучевое лечение по поводу опухолей ЦНС в детстве $(n=41)$ и во взрослом возрасте (n=69), наблюдавшихся в течение 14,5 года, не было отмечено увеличения частоты вторичных неоплазий у тех, кто лечился препаратами рГР (медиана длительности лечения 8 лет) [52]. Подобные результаты получены и в другом исследовании, проведенном в 2015 г. E. Brignardello и соавт. [53] (см. табл. 2). У пациентов с внегипофизарными опухолями головного мозга, получавших препараты рГР во взрослом возрасте $(\mathrm{n}=60$, 23 пациента получали рГР в детстве), не выявлено связи между терапией рГР и развитием вторичных неоплазий (медиана наблюдения - 17,4 года) [54].

В исследовании W. Leung [46], включавшем 910 пациентов после терапии острого лимфобластного лейкоза, у 47 человек, получивших терапию препаратами рГР, не выявлено связи между развитием вторичных опухолей и лечением ГР после 11-летнего периода наблюдения.

Следует сказать, что ряд исследователей имеют противоположное мнение. Однако влияние ГР на риск вторичных опухолей, вероятнее всего, снижается по мере увеличения продолжительности периода наблюдения. Поэтому связь между назначением ГР в детстве и риском вторичных неоплазий в отдаленном периоде после завершения лечения остается неубедительной.

В начале 1980-х гг. S. Shalet и соавт. первыми начали применять рГР для лечения радиоиндуцированного дефицита ГР. По некоторым данным, препараты ГР назначают примерно через 2 года стойкой ремиссии, однако четкий временной промежуток между окончанием противоопухолевой терапии и назначением ГР не оговорен ни в одном исследовании. Авторы клинических рекомендаций Педиатрического эндокринологического сообщества США [55] предполагают, что 1 год - это достаточный срок для того, чтобы убедиться в отсутствии раннего рецидива опухоли. Данные ретроспективных исследований свидетельствуют об отсутствии разницы в риске рецидива опухоли у пациентов после лечения медуллобластомы, получавших терапию ГР, независимо от того, прошел ли 1 год после окончания химиолучевого лечения, или 2, или 3 [45]. Следует отметить, что рецидив ЗНО возможен спустя 1 год после завершения противоопухолевой терапии как на фоне лечения препаратами
рГР, так и без него. При рассмотрении терапии рГР необходимо учитывать хронологический и костный возраст ребенка, стадию полового развития, степень задержки роста, тип опухоли, степень тяжести заболевания, общий прогноз, а также риск рецидива.

При проведении заместительной терапии рГР у детей обычно применяют дозы 0,08-0,1 мг/кг массы тела в сутки (0,2 мг/кг в неделю). По данным ряда исследований, лечение детей, перенесших ПХТ и КСО, более высокими дозами - 0,12 мг/кг в сутки (0,3-0,35 мг/кг в неделю) может улучшить ростовые показатели.

С развитием онкологических заболеваний ассоциирован высокий уровень ИФР-1. Согласно метаанализу, посвященному влиянию ИФР-1 на развитие онкологической патологии, его высокий уровень способствуют увеличению риска рака предстательной и молочных желез [56]. В этой связи во время терапии рГР контроль ИФР-1 и ИФРСБ-3 крайне важен.

Длительное время недостаточность ГР рассматривали исключительно как патологию детского возраста. Однако на сегодняшний день существуют убедительные доказательства того, что и у взрослых ДГР значим и приводит к разнообразным нарушениям: дислипидемиям (в частности, повышению уровней общего холестерина), абдоминальному ожирению, уменьшению объема тощей массы тела, снижению минеральной плотности кости, нарушению сократительной функции сердца, уменьшению мышечной силы и выносливости, нарушению сна, снижению качества жизни.

Вопрос применения ГР у взрослых, перенесших лечение $3 \mathrm{HO}$ в детстве, остается малоизученным. Несмотря на ряд опубликованных исследований об исходах терапии ДГР у детей, получивших противоопухолевую терапию, данных о продолжении применения препаратов ГР у этих пациентов во взрослом периоде, так же как и о назначении ГР после лечения $3 \mathrm{HO}$ в зрелом возрасте, до сих пор недостаточно.

S. Van den Heijkant и соавт. [57] в своем исследовании подчеркивают возможное положительное влияние препаратов ГР на индекс массы тела и состав тела у молодых взрослых после лечения острого лимфобластного лейкоза в детстве. Однако количество пациентов в данном исследовании достаточно мало $(n=20)$, поэтому его результаты не совпадают с данными другой работы [58], показавшей, что после 12-18 мес лечения препаратами ГР у пациентов, перенесших противоопухолевую терапию, улучшения в составе тела, индексе массы тела и липидном спектре были минимальными. В то же время исследователи показали улучшение качества жизни у пациентов, лечившихся ГР, что тоже немаловажно.

Больше данных о положительных и отрицательных эффектах терапии препаратами ГР накоплено у взрослых лиц с ДГР без предшествующего ЗНО. Заместительная терапия тяжелого ДГР у взрослых была одобрена в Европе в 1995 г., в США - в 1996 г. Применение препаратов ГР у взрослых лиц способствует нормализации большинства нарушений, вызываемых ДГР. Однако данная терапия не снижает общую смертность, частоту сердечнососудистых событий, а также переломов [59]. Согласно систематическому обзору литературы, данные исследований с коротким периодом наблюдения свидетельствуют об улучшении липидного обмена, а также увеличении 
минеральной плотности кости у взрослых пациентов на фоне лечения ГР [60]. Более продолжительных крупных исследований на эту тему на сегодняшний день нет, поэтому определить отдаленные эффекты терапии ГР на состояние здоровья у лиц зрелого возраста затруднительно. По данным метаанализа [61], длительная терапия препаратами ГР у взрослых не увеличивает смертность этих пациентов. Кроме того, не было показано увеличения риска онкологической патологии, роста вторичной опухоли у больных с $3 \mathrm{HO}$ в анамнезе или числа рецидивов заболевания на фоне терапии ГР.

Таким образом, несмотря на улучшение метаболических показателей на фоне терапии ГР, на сегодняшний день остается неясным влияние этого лечения на риск смертности и онкологической заболеваемости у взрослых, поэтому вопрос безопасности заместительной терапии ДГР у взрослых пациентов, перенесших химиолучевою терапию по поводу 3НО, остается открытым. Необходимо информировать пациентов о риске вероятных последствий и всех «плюсах и минусах» данной терапии и совместно принимать решение о дальнейшей тактике. Важно отметить, что лечение противопоказано лицам с активным онкологическим заболеванием и лицам с отягощенным семейным анамнезом по нейрофиброматозу 1 типа [62].

В случае положительного решения лечение препаратами ГР у взрослых лиц рекомендуется начинать не ранее чем через 2 года стойкой ремиссии [63].

Согласно зарубежным клиническим рекомендациям по диагностике и лечению ДГР у взрослых [10, 20], терапию рГР начинают с низких доз и независимо от веса, затем дозу постепенно увеличивают, ориентируясь при этом на уровень ИФР-1 и наличие/отсутствие побочных эффектов (скованность суставов, артралгии, миалгии, парестезии, задержка жидкости) (табл. 3). Целевой уровень ИФР-1 на фоне терапии рГР — середина референсного диапазона (50-й перцентиль, или 0 SDS). В тексте рекомендаций говорится о том, что низкие дозы рГР (0,1-0,2 мг/сут) представляются более безопасными у пациентов с диабетом, ожирением, а также у лиц, имеющих в анамнезе гестационный сахарный диабет. У некоторых взрослых пациентов с диагностированным в детстве ДГР, имеющих низкий ИФР-1 до лечения, могут развиваться побочные эффекты на фоне введения высоких доз ГР. У таких пациентов может быть приемлемым достижение низконормального

Таблица 3. Заместительная терапия дефицита гормона роста у взрослых (American Association of Clinical Endocrinologists 2009) (Уровень А; наилучший уровень доказательности 1)

\section{Стартовая доза:}

- возраст <30 лет: 0,4-0,5 мг/сут (доза может быть выше у пациентов, которые в детстве лечились препаратами ГР);

- возраст 30-60 лет: 0,2-0,3 мг/сут;

- возраст >60 лет: 0,1-0,2 мг/сут.

Пациентам с сахарным диабетом или нарушенной толерантностью к глюкозе показаны низкие дозы ГР $(0,1-0,2 \mathrm{мг/сут)}$.

- Подбор дозы: через 1-2 мес можно увеличивать дозу на 0,1-0,2 мг/сут под контролем уровня ИФР-1, наличия побочных эффектов, при нарушениях углеводного обмена решение данного вопроса в индивидуальном порядке. Более длительный промежуток времени и меньшие дозы могут потребоваться у пожилых пациентов.

- Ц Целевые показатели: Уровень ИФР-1 следует поддерживать в середине референсного диапазона для данного пола и возраста, если нет выраженных побочных эффектов. Возможна пробная терапия более высокими дозами, чтобы определить, насколько она эффективна и полезна. При этом ИФР-1 следует поддерживать в нормальном диапазоне и у пациента не должно быть побочных эффектов.

- Наблюдение: Динамическую оценку объективного статуса, оценку наличия побочных эффектов, уровня ИФР-1 и глюкозы плазмы натощак следует проводить с интервалом 6 мес после того, как пациент начал получать рекомендованную терапевтическую дозу. Липидный спектр следует оценивать 1 раз в год. Оценка качества жизни может проводиться 1 раз в 6-12 мес. В случае, если исходно результаты двухэнергетической рентгеновской абсорбциометрии отличались от нормы, рекомендовано повторить исследование с 2-3-летним интервалом. При наличии микроаденомы гипофиза, а также остаточной опухоли гипофиза рекомендовано периодическое выполнение МРТ головного мозга. Пациентам, получающим заместительную терапию по поводу гипотиреоза, гипокортицизма, гипогонадизма, может потребоваться коррекция дозы препаратов после начала терапии ГР.

- Особенные ситуации: Важно провести повторную диагностику дефицита ГР у пациентов, переходящих из детской во взрослую практику, в особенности у пациентов с изолированным дефицитом ГР. Данный вопрос следует решить в кратчайшие сроки, чтобы избежать длительного перерыва в терапии ГР.

- Длительность терапии ГР: Продолжительность терапии ГР не установлена. В случае, если лечение оказывает благоприятный эффект на состояние пациента, то его следует продолжать. В ситуации, когда терапевтические цели не достигнуты в течение хотя бы 2 лет, можно рассмотреть отмену терапии. Если пациент принимает решение об отмене лечения, следует рекомендовать полугодовое врачебное наблюдение, так как значительное количество пациентов могут пожелать возобновить терапию в связи с тем, что на фоне нее чувствовали себя лучше.

Примечание: ГР — гормон роста; ИФР-1 - инсулиноподобный фактор роста-1; МРТ — магнитно-резонансная томография. 
ИФР-1. На фоне лечения ДГР ИФР-1 следует контролировать с интервалом 6 мес. Остеоденситометрию следует провести до лечения, при отклонении результата от нормы рекомендовано выполнять динамические исследования каждые 2 года, оценку качества жизни - перед лечением и 1 раз в год на фоне терапии.

На сегодняшний день до конца неясно, должна ли терапия рГР быть пожизненной. Исходя из вышеупомянутых алгоритмов диагностики и лечения недостаточности ГР у взрослых $[19,20]$, при достижении терапевтических целей, таких как снижение кардиоваскулярных рисков, ИМТ, увеличение толерантности к физическим нагрузкам, а также улучшение качества жизни, прекращать терапию рГР нет повода. С другой стороны, при отсутствии описанных улучшений на фоне терапии в течение хотя бы 1-2 лет возможно решение вопроса об отмене лечения рГР.

\section{ВЗГЛЯД В БУДУЩЕЕ}

В настоящее время активно изучаются новые пролонгированные формы препаратов ГР, применяемые 1 раз в неделю или 1 раз в месяц [64], которые, вероятно, обладают большей клинической эффективностью и потенциально способны улучшать приверженность пациентов к лечению. Ввиду того, что пролонгированные формы имеют другие фармакодинамику и фармакокинетику в отличие от препаратов ГР для ежедневного введения и ГР циркулирует в крови в течение более длительного периода, вопросы безопасности применения этих препаратов относительно развития злокачественных опухолей в отдаленном периоде требуют тщательного изучения.

\section{ЗАКЛЮЧЕНИЕ}

ДГР - одно из самых распространенных эндокринных нарушений у пациентов, перенесших противоопухолевую терапию в детском возрасте, наибольший вклад в развитие которой вносит ЛТ, в частности КО. Имеющиеся на сегодняшний день данные о безопасности и эффективности заместительной терапии рГР в основном получены из обсервационных исследований, которым присущи смещенная выборка и значительные методологические ограничения. Для наиболее оптимальной оценки эффективности, потенциальных рисков развития рецидива злокачественных новообразований, а также роста вторичных опухолей у пациентов, перенесших лечение онкологиче- ских заболеваний, на фоне заместительной терапии ГР необходимы тщательно спланированные проспективные рандомизированные плацебо-контролируемые исследования. Однако подобные исследования неэтичны, в частности среди детей, поскольку часть пациентов не будут получать лечение, несмотря на диагностированный ДГР и необходимость его восполнения.

Убедительных доказательств увеличения риска рецидива 3 НО у пациентов, перенесших онкологическое заболевание в детстве, на фоне лечения препаратами ГР на сегодняшний день нет. Однако данная терапия может потенциально повышать риск вторичных неоплазий. Подобная терапия показана детям с недостаточностью ГР для достижения нормального роста. В то же время, несмотря на улучшение метаболических показателей на фоне лечения, вопрос его назначения взрослым на сегодняшний день до конца не решен ввиду отсутствия убедительных данных о влиянии терапии ГР на такие параметры, как смертность, сердечно-сосудистые события, переломы. В свете вышеописанного, при рассмотрении терапии препаратами ГР у взрослых пациентов, перенесших онкологическое заболевание в детстве, необходимо учитывать тип перенесенной опухоли, степень тяжести заболевания, общий прогноз, риск рецидива. Важно обсуждать совместно с пациентами и их семьями возможности такого лечения, информировать о положительных сторонах терапии и о потенциальных рисках и совместно принимать решение о дальнейшей тактике.

\section{ДОПОЛНИТЕЛЬНАЯ ИНФОРМАЦИЯ}

Источники финансирования. Работа выполнена по инициативе авторов без привлечения финансирования.

Конфликт интересов. Авторы декларируют отсутствие явных и потенциальных конфликтов интересов, связанных с содержанием настоящей статьи.

Участие авторов. Павлова М.Г., Целовальникова Т.Ю. - концепция и дизайн исследования, подготовка и написание текста статьи; Юдина А.Е., Голоунина О.О. - анализ данных, подготовка текста статьи; Зилов А.В., Мазеркина Н.А., Желудкова О.Г. - внесение в рукопись существенной правки с целью повышения научной ценности статьи; Фадеев В.В. - внесение в рукопись существенной правки, одобрение финальной версии рукописи. Все авторы одобрили финальную версию статьи перед публикацией, выразили согласие нести ответственность за все аспекты работы, подразумевающую надлежащее изучение и решение вопросов, связанных с точностью или добросовестностью любой части работы.

\section{СПИСОК ЛИТЕРАТУРЫ | REFERENCES}

American Cancer Society. Cancer Facts \& Figures, 2018. Cancer Statistics. 2018;2018:23.

2. Каприн А.Д., Старинский В.В., Шахзадова А.О. Злокачественные новообразования в России в 2019 году (заболеваемость и смертность). - М.: МНИОИ им. П.А. Герцена — филиал ФГБУ НМИРЦ. Минздрава России; 2020. 252 c. [Kaprin AD, Starinskii W, Shakhzadova AO. Zlokachestvennye novoobrazovaniya v Rossii v 2019 godu (zabolevaemost' i smertnost'). Moscow: MNIOI im. P.A. Gertsena filial FGBU NMIRTs. Minzdrava Rossii; 2020. 252 p. (In Russ.)].

3. Желудкова О.Г., Поляков В.Г., Рыков М.Ю., и др. Клинические проявления онкологических заболеваний у детей: практические рекомендачии / Под ред. В.Г. Полякова, М.Ю. Рыкова. - СПб.: Типография Михаила Фурсова; 2017. 52 c. [Zheludkova OG, Polyakov VG, Rykov MYu, et al. Klinicheskie proyavleniya onkologicheskikh zabolevaniĨ u deteǐ: prakticheskie rekomendatsii. Ed. by Polyakova VG, Rykova MYu. — SPb.: Tipografiya Mikhaila Fursova; 2017. 52 p. (In Russ.)].

4. Gurney JG, Kadan-Lottick NS, Packer RJ, et al. Endocrine and cardiovascular late effects among adult survivors of childhood brain tumors: Childhood Cancer Survivor Study. Cancer. 2003;97:663-673. doi: https://doi.org/10.1002/cncr.11095

5. Боброва Е.И., Павлова М.Г., Сотников В.М., и др. Гипопитуитаризм после облучения гипоталамо-гипофизарной системы // Клиническая и экспериментальная тиреоидология. - 2013. T. 9. - №3. - C. 15-20. [Bobrova El, Pavlova MG, Sotnikov VM, et al. Hypopituitarism after radiotherapy for childhood malignant tumors. Clinical and experimental thyroidology. 2013;9(3):15-20. (In Russ.)]. doi: https://doi.org/10.14341/ket20139315-20 
6. Uday S, Murray RD, Picton S, et al. Endocrine sequelae beyond 10 years in survivors of medulloblastoma. Clin Endocrinol (Oxf). 2015;83(5):663-670. doi: https://doi.org/10.1111/cen.12815

7. Mirouliaei M, Shabani M, Bakhshi F, Ordouei M. Radiationinduced hypopituitarism in children with acute lymphoblastic leukemia. Indian J Med Paediatr Oncol. 2013;34(1):8-10. doi: https://doi.org/10.4103/0971-5851.113396

8. Olshan JS, Gubernick J, Packer RJ, et al. The effects of adjuvant chemotherapy on growth in children with medulloblastoma. Cancer. 1992;70(7):2013-2017. doi: https://doi.org/10.1002/10970142(19921001)70:7<2013::AID-CNCR2820700734>3.0.CO;2-J

9. Павлова М.Г., Сыч Ю.П., Боброва Е.И., и др. Эндокринные последствия комплексного лечения медуллобластом в детском и молодом возрасте / Сборник тезисов IV (XXVII) Национального конгресса эндокринологов с международным участием. - M.; 2021. — 202 c. [Pavlova MG, Sych YuP, Bobrova El, et al. Endokrinnye posledstviya kompleksnogo lecheniya medulloblastom $v$ detskom i molodom vozraste. In: Sbornik tezisov IV (XXVII) Natsional'nogo kongressa endokrinologov s mezhdunarodnym uchastiem. Moscow; 2021. 202 p. (In Russ.)]. doi: https://doi.org/10.14341/Conf22-25.09.21-202

10. Jarfelt $M$, Bjarnason $R$, Lannering B. Young adult survivors of childhood acute lymphoblastic leukemia: spontaneous GH secretion in relation to CNS radiation. Pediatr Blood Cancer. 2004:42:582-588. doi: https://doi.org/10.1002/pbc.20020

11. Brignardello E, Felicetti F, Castiglione A, et al. Endocrine health conditions in adult survivors of childhood cancer: the need for specialized adult-focused follow-up clinics. Eur J Endocrinol. 2013;168(3):465-472. doi: https://doi.org/10.1530/EJE-12-1043

12. Darzy KH. Radiation-induced hypopituitarism. Curr Opin Endocrinol Diabetes Obes. 2013;20(4):342-353. doi: https://doi.org/10.1097/MED.0b013e3283631820

13. Darzy KH, Pezzoli SS, Thorner MO, Shalet SM. The dynamics of growth hormone $(\mathrm{GH})$ secretion in adult cancer survivors with severe GH deficiency acquired after brain irradiation in childhood for nonpituitary brain tumors: evidence for preserved pulsatility and diurnal variation with increased secretor. J Clin Endocrinol Metab. 2005;90(5):2794-2803. doi: https://doi.org/10.1210/jc.2004-2002

14. Brauner R, Czernichow P, Rappaport R. Greater susceptibility to hypothalamopituitary irradiation in younger children with acute lymphoblastic leukemia. J Pediatr. 1986;108(2):332. doi: https://doi.org/10.1016/s0022-3476(86)81027-7

15. Samaan NA, Schultz PN, Yang KPP, et al. Endocrine complications after radiotherapy for tumors of the head and neck. J Lab Clin Med. 1987;109:364-372. doi: https://doi.org/10.5555/uri:pii:002221438790059X

16. Shen Y, Zhang J, Zhao Y, et al. Diagnostic value of serum IGF-1 and IGFBP-3 in growth hormone deficiency: a systematic review with meta-analysis. Eur J Pediatr. 2015;174(4):419-427. doi: https://doi.org/10.1007/s00431-014-2406-3

17. Cattoni A, Clarke E, Albanese A. The Predictive Value of InsulinLike Growth Factor 1 in Irradiation-Dependent Growth Hormone Deficiency in Childhood Cancer Survivors. Horm Res Paediatr. 2018;90(5):314-325. doi: https://doi.org/10.1159/000495760

18. Tavares ABW, Collett-Solberg PF. Growth hormone deficiency and the transition from pediatric to adult care. J Pediatr (Rio J). 2021;97(6):595-602. doi: https://doi.org/10.1016/j.jped.2021.02.007

19. Yuen KCJ, Biller BMK, Radovick S, et al. American association of clinical endocrinologists and American college of endocrinology guidelines for management of growth hormone defficiency in adults and patients transitioning from pediatric to adult care. Endocr Pract. 2019;25(11):1191-1232. doi: https://doi.org/10.4158/GL-2019-0405

20. Molitch ME, Clemmons DR, Malozowski S, et al. Evaluation and treatment of adult growth hormone deficiency: an Endocrine Society clinical practice guideline. J Clin Endocrinol Metab. 2011;96(6):1587-1609. doi: https://doi.org/10.1210/jc.2011-0179

21. Cook DM, Yuen KC, Biller BM, et al. American Association of Clinical Endocrinologists medical guidelines for clinical practice for growth hormone use in growth hormone-deficient adults and transition patients - 2009 update. Endocr Pract. 2009;15(S2):1-29. doi: https://doi.org/10.4158/EP.15.S2.1

22. Bidlingmaier M. Problems with $\mathrm{GH}$ assays and strategies toward standardization. Eur J Endocrinol. 2008;159(S1):S41-44. doi: https://doi.org/10.1530/EJE-08-0284
23. Hymer WC, Kennett MJ, Maji SK, et al. Bioactive growth hormone in humans: Controversies, complexities and concepts. Growth Horm IGF Res. 2020;50:9-22. doi: https://doi.org/10.1016/j.ghir.2019.11.003

24. Bidlingmaier M, Freda PU. Measurement of human growth hormone by immunoassays: current status, unsolved problems and clinical consequences. Growth Horm IGF Res. 2010;20(1):19-25. doi: https://doi.org/10.1016/j.ghir.2009.09.005

25. Baumann G. Growth hormone binding protein 2001. J Pediatr Endocrinol Metab. 2001;14(4):355-375. doi: https://doi.org/10.1515/jpem.2001.14.4.355

26. Tanaka T, Tachibana K, Shimatsu A, et al. A nationwide attempt to standardize growth hormone assays. Horm Res. 2005;64(S2):6-11. doi: https://doi.org/10.1159/000087746

27. Binder G. Growth hormone deficiency: new approaches to the diagnosis. Pediatr Endocrinol Rev. 2011;9(S1):535-537.

28. Dall R, Longobardi S, Ehrnborg C, et al. Growth Hormone Research Society. Consensus guidelines for the diagnosis and treatment of growth hormone (GH) deficiency in childhood and adolescence: summary statement of the GH Research Society. GH Research Society. J Clin Endocrinol Metab. 2000;85(11):3990-3993. doi: https://doi.org/10.1210/jcem.85.11.6984

29. Trainer PJ. Consensus statement on the standardisation of GH assays. Eur JEndocrinol. 2006;155(1):1-2. doi: https://doi.org/10.1530/eje.1.02186

30. Hoeck HC, Vestergaard P, Jakobsen PE, et al. Diagnosis of growth hormone $(\mathrm{GH})$ deficiency in adults with hypothalamic-pituitary disorders: comparison of test results using pyridostigmine plus $\mathrm{GH}$-releasing hormone (GHRH), clonidine plus GHRH, and insulininduced hypoglycemia as GH secretagogues. J Clin Endocrinol Metab. 2000;85(4):1467-1472. doi: https://doi.org/10.1210/jcem.85.4.6538

31. Markkanen HM, Pekkarinen T, Välimäki MJ, et al. Comparison of two growth hormone stimulation tests and their cut-off limits in healthy adults at an outpatient clinic. Growth Horm IGF Res. 2013;23(5):165-169. doi: https://doi.org/10.1016/j.ghir.2013.06.002

32. Poyrazoğlu Ş, Akçay T, Arslanoğlu I, et al. Current Practice in Diagnosis and Treatment of Growth Hormone Deficiency in Childhood: A Survey from Turkey. J Clin Res Pediatr Endocrinol. 2015;7(1):37-44 doi: https://doi.org/10.4274/jcrpe.1794

33. Biller BMK, Samuels MH, Zagar A, et al. Sensitivity and specificity of six tests for the diagnosis of adult GH deficiency. J Clin Endocrinol Metab. 2002;87(5):2067-2079. doi: https://doi.org/10.1210/jcem.87.5.8509

34. Deutschbein T, Bidlingmaier M, Schopohl J, et al. Anthropometric factors have significant influence on the outcome of the GHRH-arginine test — establishment of normative data for an automated immunoassay specifically measuring $22 \mathrm{kD}$ human growth hormone. Eur J Endocrinol. 2017;176(3):273-281. doi: https://doi.org/10.1530/EJE-16-0668

35. Conceição FL, da Costa e Silva A, Leal Costa AJ, Vaisman M. Glucagon stimulation test for the diagnosis of GH deficiency in adults. J Endocrinol Invest. 2003;26(11):1065-1070. doi: https://doi.org/10.1007/BF03345251

36. Gómez JM, Espadero RM, Escobar-Jiménez F, et al. Growth hormone release after glucagon as a reliable test of growth hormone assessment in adults. Clin Endocrinol (Oxf). 2002;56(3):329-334. doi: https://doi.org/10.1046/j.1365-2265.2002.01472.x

37. Aimaretti G, Corneli $G$, Razzore P, et al. Comparison between insulin-induced hypoglycemia and growth hormone $(\mathrm{GH})$ - releasing hormone + arginine as provocative tests for the diagnosis of $\mathrm{GH}$ deficiency in adults. J Clin Endocrinol Metab. 1998;83(5):1615-1618. doi: https://doi.org/10.1210/jcem.83.5.4837

38. Дедов И.И., Мельниченко Г.А. Эндокринология. Национальное Руководство. 2-е изд.. - М.: ГЭОТАР-Медиа, 2018. - 832 с. [Dedov II, Mel'nichenko GA. Endokrinologiya. Natsional'noe Rukovodstvo. 2-elzd. Moscow: GEOTAR-Media, 2018. 832 p. (In Russ.)].

39. Голоунина О.О., Павлова М.Г., Белая Ж.Е., и др. Функциональное состояние эндокринной системы и минеральная плотность костной ткани в отдаленном периоде после комбинированного лечения злокачественных опухолей головного мозга в детском и молодом возрасте // Проблемы эндокринологии. - 2021. T. 67. — №1. - C. 31-40. [Golounina OO, Pavlova MG, Belaya ZE, et al. Endocrine late-effects and bone mineral density after combined treatment of malignant brain tumors. Problems of Endocrinology. 2021;67(1):31-40. (In Russ.)]. doi: https://doi.org/10.14341/probl12680

40. Chae HW, Kim DH, Kim HS. Growth hormone treatment and risk of malignancy. Korean J Pediatr. 2015;58(2):41-46. doi: https://doi.org/10.3345/kjp.2015.58.2.41 
41. Robison LL, Mertens AC, Boice JD, et al. Study design and cohort characteristics of the Childhood Cancer Survivor Study: A multi-institutional collaborative project. Med Pediatr Oncol. 2002;38(4):229-239. doi: https://doi.org/10.1210/jcem.83.5.4837

42. Sklar CA, Mertens AC, Mitby P, et al. Risk of disease recurrence and second neoplasms in survivors of childhood cancer treated with growth hormone: a report from the Childhood Cancer Survivor Study. J Clin Endocrinol Metab. 2002;87(7):3136-3141. doi: https://doi.org/10.1210/jcem.87.7.8606

43. Arslanian SA, Becker DJ, Lee PA, et al. Growth hormone therapy and tumor recurrence. Findings in children with brain neoplasms and hypopituitarism. Am J Dis Child. 1985;139(4):347-350 doi: https://doi.org/10.1001/archpedi.1985.02140060029020

44. Chae HW, Park YS, Kim DS, et al. Final height and insulin-like growth factor-1 in children with medulloblastoma treated with growth hormone. Child's Nerv Syst. 2013;29(10):1859-1863. doi: https://doi.org/10.1007/s00381-013-2124-6

45. Packer RJ, Boyett JM, Janssa J, et al. Growth hormone replacement therapy in children with medulloblastoma: use and effect on tumor control. J Clin Oncol. 2001;19(2):480-487. doi: https://doi.org/10.1200/JCO.2001.19.2.480

46. Leung W, Rose SR, Zhou Y, et al. Outcomes of growth hormone replacement therapy in survivors of childhood acute lymphoblastic leukemia. J Clin Oncol. 2002;20(13):2959-2964. doi: https://doi.org/10.1200/JCO.2002.09.142

47. Carel J-C, Butler G. Safety of recombinant human growth hormone Endocr Dev. 2010;18:40-54. doi: https://doi.org/10.1159/000316126

48. Friedman $\mathrm{DL}$, Whitton J, Leisenring $\mathrm{W}$, et al. Subsequent neoplasms in 5-year survivors of childhood cancer: The childhood cancer survivor study. J Nat/ Cancer Inst. 2010;102(14):1083-1095. doi: https://doi.org/10.1093/jnci/djq238

49. Ergun-Longmire B, Mertens AC, Mitby P, et al. Growth hormone treatment and risk of second neoplasms in the childhood cancer survivor. J Clin Endocrinol Metab. 2006;91(9):3494-3498. doi: https://doi.org/10.1210/jc.2006-0656

50. Patterson BC, Chen Y, Sklar CA, et al. Growth Hormone Exposure as a Risk Factor for the Development of Subsequent Neoplasms of the Central Nervous System: A Report From the Childhood Cancer Survivor Study. J Clin Endocrinol Metab. 2014;99(6):2030-2037. doi: https://doi.org/10.1210/jc.2013-4159

51. Мазеркина Н.А., Горелышев С.К., Желудкова О.Г., и др. Результаты заместительной терапии гормоном роста у детей с опухолями головного мозга // Проблемы Эндокринологии. 2013. - T. 59. — №2. — C. 19-25. [Mazerkina NA, Gorelyshev SK, Zheludkova OG, et al. Results of growth hormone substitution therapy in children with brain tumours. Problems of Endocrinology. 2013;59(2):19-25. (In Russ.)]. doi: https://doi.org/10.14341/probl201359219-25

52. Mackenzie S, Craven T, Gattamaneni HR, et al. Longterm safety of growth hormone replacement after CNS irradiation. J Clin Endocrinol Metab. 2011:96(9):2756-2761. doi: https://doi.org/10.1210/jc.2011-0112

53. Brignardello E, Felicetti F, Castiglione A, et al. GH replacement therapy and second neoplasms in adult survivors of childhood cancer: A retrospective study from a single institution. J Endocrinol Invest. 2015;38(2):171-176. doi: https://doi.org/10.1007/s40618-014-0179-1

54. Jostel A, Mukherjee A, Hulse PA, Shalet SM. Adult growth hormone replacement therapy and neuroimaging surveillance in brain tumour survivors. Clin Endocrinol (Oxf). 2005;62(6):698-705. doi: https://doi.org/10.1111/j.1365-2265.2005.02282.x

55. Wilson TA, Rose SR, Cohen $\mathrm{P}$, et al. Update of guidelines for the use of growth hormone in children: the Lawson Wilkins Pediatric Endocrinology Society Drug and Therapeutics Committee. J Pediatr. 2003;143(4):415-421. doi: https://doi.org/10.1067/s0022-3476(03)00246-4

56. Renehan AG, Zwahlen M, Minder C, et al. Insulin-like growth factor (IGF)-I, IGF binding protein-3, and cancer risk: systematic review and metaregression analysis. Lancet (London, England). 2004;363(9418):1346-1353. doi: https://doi.org/10.1016/S0140-6736(04)16044-3

57. van den Heijkant S, Hoorweg-Nijman G, Huisman J, et al. Effects of growth hormone therapy on bone mass, metabolic balance, and well-being in young adult survivors of childhood acute lymphoblastic leukemia. J Pediatr Hematol Oncol. 2011;33(6):e231-238. doi: https://doi.org/10.1097/MPH.0b013e31821bbe7a

58. Murray RD, Darzy KH, Gleeson HK, Shalet SM. GH-deficient survivors of childhood cancer: GH replacement during adult life. J Clin Endocrinol Metab. 2002;87(1):129-135. doi: https://doi.org/10.1210/jcem.87.1.8146

59. Höybye C, Beck-Peccoz P, Simsek S, et al. Safety of current recombinant human growth hormone treatments for adults with growth hormone deficiency and unmet needs. Expert Opin Drug Saf. 2020;19(12):1539-1548. doi: https://doi.org/10.1080/14740338.2020.1839410

60. Ahmid M, Perry CG, Ahmed SF, Shaikh MG. Growth hormone deficiency during young adulthood and the benefits of growth hormone replacement. Endocr Connect. 2016;5(3):R1-R11. doi: https://doi.org/10.1530/EC-16-0024

61. Stochholm K, Johannsson G. Reviewing the safety of $\mathrm{GH}$ replacement therapy in adults. Growth Horm IGF Res. 2015;25(4):149-157. doi: https://doi.org/10.1016/j.ghir.2015.06.006

62. Schiffman JD, Geller Jl, Mundt E, et al. Update on pediatric cancer predisposition syndromes. Pediatr Blood Cancer. 2013:60(8):1247-1252. doi: https://doi.org/10.1002/pbc.24555

63. Yuen KCJ, Heaney AP, Popovic V. Considering GH replacement for $\mathrm{GH}$-deficient adults with a previous history of cancer: a conundrum for the clinician. Endocrine. 2016;52(2):194-205. doi: https://doi.org/10.1007/s12020-015-0840-2

64. Høybye C, Cohen P, Hoffman AR, et al. Christiansen JS. Status of long-acting-growth hormone preparations - 2015. Growth Hormone and IGF Research. 2015;25(5):201-206. doi: https://doi.org/10.1016/j.ghir.2015.07.004

\section{ИНФОРМАЦИЯ ОБ АВТОРАХ [AUTHORS INFO]:}

*Павлова Мария Геннадиевна, к.м.н. [Maria G. Pavlova, MD, PhD]; адрес: Россия, 119991, Москва, ул. Трубецкая, д. 8, стр. 2 [address: 8-2 Trubetskaya street, 119991 Moscow, Russia]; ORCID: http://orcid.org/0000-0001-6073-328X; eLibrary SPIN: 2205-1288; e-mail: mgp.med@gmail.com

Целовальникова Татьяна Юрьевна, к.м.н. [Tatiana Y. Tselovalnikova, MD, PhD]; ORCID: http://orcid.org/0000-0003-4262-2724; eLibrary SPIN: 2688-6494; e-mail: t.tselovalnikova@gmail.com Юдина Алла Евгеньевна, к.м.н. [Alla E. Yudina, MD, PhD]; ORCID: https://orcid.org/0000-0002-4704-8453; eLibrary SPIN: 9307-7889; e-mail: alla1301@yandex.ru

Голоунина Ольга Олеговна, студентка [Olga O. Golounina, student]; ORCID: https://orcid.org/0000-0003-2320-1051; eLibrary SPIN: 7793-2123; e-mail: olga.golounina@mail.ru

Зилов Алексей Вадимович, к.M.н. [Alexey V. Zilov, MD, PhD]; ORCID: https://orcid.org/0000-0003-3494-8011; eLibrary SPIN: 8575-1247; e-mail: avzilov@hotmail.com

Мазеркина Надежда Александровна, д.м.н. [Nadezhda A. Mazerkina, MD, PhD];

ORCID: https://orcid.org/0000-0002-0428-0498; eLibrary SPIN:1012-2923; e-mail: nmazer@nsi.ru

Желудкова Ольга Григорьевна, д.м.н., професcop [Olga G. Zheludkova, MD, PhD, Professor]; ORCID: https://orcid.org/0000-0002-8607-3635; eLibrary SPIN: 4850-7788; e-mail: clelud@mail.ru

Фадеев Валентин Викторович, д.м.н., профессор, член-корр. PAH [Valentin V. Fadeyev, MD, PhD, Professor]; ORCID: http://orcid.org/0000-0002-3026-6315; eLibrary SPIN: 6825-8417; e-mail: walfad@mail.ru

*Автор, ответственный за переписку / Corresponding author. 


\section{ЦИТИРОВАТЬ:}

Павлова М.Г., Целовальникова Т.Ю., Юдина А.Е., Голоунина О.О., Зилов А.В., Мазеркина Н.А., Желудкова О.Г., Фадеев В.В. Дефицит гормона роста у лиц, перенесших химиолучевую терапию злокачественных опухолей головного мозга и острого лимфобластного лейкоза в детстве // Ожирение и метаболизм. — 2021. — Т. 18. — №4. — С. 484-495. doi: https://doi.org/10.14341/omet12786

\section{TO CITE THIS ARTICLE:}

Pavlova MG, Tselovalnikova TY, Yudina AE, Golounina OO, Zilov AV, Mazerkina NA, Zheludkova OG, Fadeev VV. Growth hormone deficiency in childhood brain tumors and acute lymphoblastic leukemia survivors. Obesity and metabolism. 2021;18(4):484-495. doi: https://doi.org/10.14341/omet12786 